\title{
TU/e EmonOWEN

\section{Effect of disorder strength on the fracture pattern in heterogeneous networks}

\section{Citation for published version (APA):}

Malakhovsky, I., \& Michels, M. A. J. (2007). Effect of disorder strength on the fracture pattern in heterogeneous networks. Physical Review B, 76(14), 144201-1/3. [144201]. https://doi.org/10.1103/PhysRevB.76.144201

DOI:

10.1103/PhysRevB.76.144201

Document status and date:

Published: 01/01/2007

\section{Document Version:}

Publisher's PDF, also known as Version of Record (includes final page, issue and volume numbers)

\section{Please check the document version of this publication:}

- A submitted manuscript is the version of the article upon submission and before peer-review. There can be important differences between the submitted version and the official published version of record. People interested in the research are advised to contact the author for the final version of the publication, or visit the $\mathrm{DOI}$ to the publisher's website.

- The final author version and the galley proof are versions of the publication after peer review.

- The final published version features the final layout of the paper including the volume, issue and page numbers.

Link to publication

\section{General rights}

Copyright and moral rights for the publications made accessible in the public portal are retained by the authors and/or other copyright owners and it is a condition of accessing publications that users recognise and abide by the legal requirements associated with these rights.

- Users may download and print one copy of any publication from the public portal for the purpose of private study or research.

- You may not further distribute the material or use it for any profit-making activity or commercial gain

- You may freely distribute the URL identifying the publication in the public portal.

If the publication is distributed under the terms of Article 25fa of the Dutch Copyright Act, indicated by the "Taverne" license above, please follow below link for the End User Agreement:

www.tue.nl/taverne

Take down policy

If you believe that this document breaches copyright please contact us at:

openaccess@tue.nl

providing details and we will investigate your claim. 


\title{
Effect of disorder strength on the fracture pattern in heterogeneous networks
}

\author{
I. Malakhovsky* and M. A. J. Michels \\ Group Polymer Physics, Technische Universiteit Eindhoven, P.O. Box 513, 5600 MB Eindhoven, The Netherlands
}

(Received 25 July 2006; revised manuscript received 12 June 2007; published 2 October 2007)

\begin{abstract}
We present simulation results on fracture and random damage percolation in disordered two-dimensional (2D) lattices of different sizes. We systematically study the effect of disorder strength on the stress-strain behavior and on the evolving fracture pattern. In particular, the similarity of damage-cluster statistics between fracture and random percolation is investigated. For fracture in highly disordered systems, we confirm and extend our earlier results on the existence of a percolationlike damage regime, with accurate scaling laws for the cluster statistics, but we show that this regime vanishes at intermediate disorder strength. For low disorder, a qualitatively different and anisotropic damage pattern develops from the very beginning of loading. Both for low and high disorder strengths, macroscopic localization and strong damage anisotropy set in around the maximum-stress point, leading to the final crack formation. The surface roughness of the ultimate crack shows accurate size scaling, with a universal roughness exponent independent of the disorder strength but slightly dependent on the precise definition of the crack profile. The simulated roughness exponent is in good agreement with other numerical and experimental results on 2D systems and also close to the prediction of gradient percolation.
\end{abstract}

DOI: 10.1103/PhysRevB.76.144201 PACS number(s): 62.20.Mk, 64.60.Ak, 89.75.Da, 61.43.Bn

\section{INTRODUCTION}

Fracture is a phenomenon for which disorder undoubtedly plays an important role. If high enough, the microscopic disorder of materials can sufficiently influence their macroscopic properties and the way they fail. For example, the dependence of material strength on sample size is due to disorder and has been observed and analyzed from the beginning of material science. ${ }^{1}$ Besides the theoretical significance of this phenomenon, it is obviously of a great practical importance.

Universal scaling of crack roughness is another important experimental fact of fracture ${ }^{2}$ in which disorder plays a role. The striking observation is that materials with very different properties and breakdown mechanisms have the same roughness-scaling law and very close (if not equal) values of roughness-scaling exponent. Understanding this universality is a fundamental challenge which has already led to much experimental $^{3-6}$ and theoretical ${ }^{2,7-10}$ research.

However, disorder is only one of many important factors in fracture, which is a very complex process that almost always combines many aspects of different nature. A clear understanding of the effect of disorder and the ability to delimit it from other aspects of fracture is therefore highly important. Fracture is a process of damage development controlled by imposed stress or strain and by material and geometrical properties. This process becomes randomized in the presence of disorder. If the extent of disorder is high, randomness can start to prevail over other aspects of failure. The situation when the process of damage development is totally random is described by the random-percolation theory. The process of random percolation of damage with a network of damaged bonds as an object of consideration should be distinguished from rigidity- and connectivity-percolation studies, ${ }^{11}$ which deal with remaining (unbroken) bonds. Moreover, in rigidity percolation, clusters are identified according to their rigidity, not connectivity. As a result of this, for rigidity percolation, the governing scaling exponents are different from the ones in random-percolation theory. In fact, it can be shown that in the limit of infinite disorder, the theoretical description of fracture, at least in the scalar version of dielectric breakdown, can be mapped onto a random-percolation problem. ${ }^{12}$ However, it is not possible to analyze this limit numerically. Contrary to fracture, random percolation (RP), being a purely mathematical subject, is rather well understood. Comparing the two phenomena can shed light upon the role of disorder in fracture and help to understand whether randompercolation arguments can be applied to materials breakdown. Apparently, the extent of similarity should depend on disorder strength. Nevertheless, the clear picture showing how the process of fracture changes depending on the disorder strength is still missing. This problem is very hard to handle theoretically. Therefore, many authors have resorted to the numerical simulation of fracture with lattice models. ${ }^{13-18}$ In particular, three types of models have been used: a fuse-lattice model, ${ }^{15,16}$ a spring-lattice model, ${ }^{17-19}$ and a spring-lattice model with bond-bending forces. ${ }^{13,20}$ The first model is nothing but a lattice of burnable fuses obeying Kirchhoff's equations, the second one is a lattice of centralforce springs with an elastic constitutive relation, and the third model is similar to the second one but also contains the effect of bending.

The first numerical studies of fracturing lattices which had focus on scaling properties and percolation aspects were made in Refs. 13 and 14 and concluded on the existence of a regime dominated by disorder and on the existence of scaling relations. From an analysis of fuse networks with strong power-law disorder, ${ }^{15}$ it was concluded that fracture with strong disorder belongs to the same universality class as RP, i.e., the two phenomena have identical scaling laws with numerically the same scaling exponents. Later, the authors of Refs. 16 and 17 came to different conclusions both for fuse networks and networks of central-force springs. To our knowledge, no study was made of the precise role of disorder 
strength in central-force lattices, though for fuse networks the first study on this matter was performed more than a decade ago. ${ }^{21}$ In an attempt to clarify the issue of similarity with RP, we have already investigated fracturing centralforce lattices with a power-law strength distribution and a high disorder strength and have compared the evolving damage pattern with that of random percolation, both theoretical and simulated. ${ }^{18}$ The validity of theoretical scaling laws of RP for fracture has been used as a measure of similarity. We have observed three clearly distinguishable regimes in the damage development, of which only the middle one is similar to RP. In the first regime, with low fracture damage, short-range localization was observed, which makes a difference with RP. Within the middle regime, the statistics of damage clusters of simulated fracture and of simulated RP revealed the same scaling properties. In the last regime, macroscopic localization and anisotropy were recognized, again making fracture different from RP. Although in the last regime of fracture the RP scaling relations proved not valid, the roughness of the final crack was found to scale very accurately, with the roughness-scaling exponent $\zeta$ $=0.65 \pm 0.07$ or $\zeta=0.74 \pm 0.11$, depending on the precise definition of the final crack path. The obtained values for the roughness-scaling exponent are consistent with the value $0.68 \pm 0.04$ that was determined experimentally for quasitwo-dimensional cracks in wood, ${ }^{3}$ and (marginally) consistent with the prediction for random percolation in a gradient, $\zeta=8 / 11=0.73 .^{15}$

This research has left an obvious question: How do these three features (the existence of three regimes, similarity between the second regime and RP, and roughness scaling) depend on the disorder strength? In this work, we present a systematic investigation of these dependencies. In particular, we check and confirm our earlier results for even higher disorder strength. Then, we systematically decrease the disorder and analyze the behavior of the fracturing lattices. Attention is paid to macroscopic properties of the process such as stress-strain dependencies, damage localization, and macroscopic features of the damage clusters (weight, spatial size, and possible anisotropy), as well as to the microscopic damage-cluster statistics and short-range localization. We also analyze the final crack formation and crack-roughness scaling.

The paper is organized in the following way. In Sec. II, we describe our model and the methods that we use for the analysis. In Sec. III, we present the main results of our simulations. Here, we first address macroscopic properties of our model. In Sec. III A, we focus on the size scaling of macroscopic stress and strain. We also illustrate here how stressstrain and stress-damage dependencies change with the disorder strength. Macroscopic features of damage clusters are presented in Sec. III B. We confirm that in the case of strong disorder, there is a range of damage density where RP scaling relations numerically hold for fracture. Then, in Secs. III C and III D, we separately describe the detailed results of fracture simulations with high and low disorder strengths. At the end of Sec. III, we focus on the damage localization

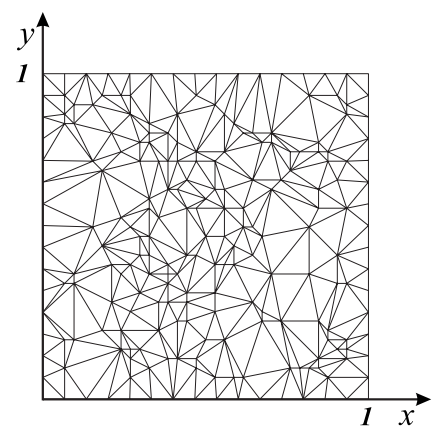

FIG. 1. Example of a Delaunay-tessellation lattice $(L=15)$ as used in the simulations.

(Sec. III E) and the scaling of crack roughness (Sec. III F). In Sec. IV, we summarize our main conclusions.

\section{MODEL AND METHODS}

Our analysis is based on the fracture simulation of twodimensional (2D) lattices of central-force springs, ${ }^{14,22}$ with power-law disorder in the critical strength of the individual springs. We compare the fracture results with the results of RP simulation on geometrically identical lattices. At the stage of postprocessing, we check the validity of scaling relations of RP in the fracture data, in order to compare the two phenomena. In the analysis, the focus is on the following features of the processes: macroscopic stress and strain and their scaling with the lattice size $L$, statistical properties of damage clusters with respect to their weight and spatial extent, damage anisotropy, and localization. We pay special attention to the crack-roughness scaling and to the behavior of the scaling exponent $\zeta$. In all these aspects, we systematically consider the dependence on the disorder strength.

The model is a 2D lattice with a random geometry, built as a Delaunay tessellation (a set of lines connecting each node to its natural neighbors ${ }^{23}$ ) of a square region with randomly distributed nodes (Fig. 1) uniquely chosen for each lattice sample. In the case of fracture the disorder is introduced via the distribution of thresholds, i.e., critical strains at which bonds break. To be in line with previous research of other authors and ourselves, we employ a power-law distribution of thresholds $t$,

$$
P(t)=(1-\alpha) w^{\alpha-1} t^{-\alpha}, \quad t \in[0, w],
$$

where the disorder strength $\alpha$ varies from 0 (uniform disorder) to 0.75 (high disorder). We artificially shift all obtained thresholds collectively from zero toward higher values by a very small number. This is done in order to preserve the integrity of lattices and to avoid numerical problems induced by thresholds that are extremely close to zero. Such thresholds are an unavoidable consequence of the details of the numerical procedure, in which randomly generated threshold values have to be converted into thresholds obeying the distribution [Eq. (1)]. Higher values of $\alpha$ are not analyzed in 
TABLE I. Statistics of simulation runs.

\begin{tabular}{lc}
\hline \hline Linear lattice size $L$ & Number of runs \\
\hline 25 & 500 \\
50 & 500 \\
100 & 100 \\
200 & 25 \\
\hline \hline
\end{tabular}

view of computational difficulties. We keep $w$ fixed, independent of $\alpha$, which means that more disordered lattices will on average be weaker. Note that the notation for the exponent in the disorder distribution can be different in other publications. For example, in Ref. 12, $\alpha$ is used instead of $-\alpha$, and in Ref. $15,-\alpha-1$ is employed. Consequently, the case of infinite disorder corresponds to $\alpha=-1$ in Ref. 12, to $\alpha=0$ in Ref. 15 , and to $\alpha=1$ in our case.

For fracture simulations, strain is applied to the lattice via imposing equal relative displacement to the opposite nodes at the left and right sides of the lattice. The opposite nodes of the upper and lower sides are restricted to have equal relative displacement, which is defined by the solution as an independent degree of freedom. ${ }^{18}$ In this way, the shapes of the deformed opposite sides always stay identical, as if the lattice were wrapped around a torus.

A bond will be broken if it has the highest ratio of actual strain and threshold and if this ratio is more than or equal to 1. Bonds are being broken one by one, and after each breaking event, the equilibrium equations are resolved again. This assumes that the redistribution of stresses in the lattice occurs faster than the breaking of bonds. Since dynamic effects are not taken into account, we can say that our model represents a quasistatic fracture.

In addition to the simulation of fracture, we also perform a simulation of random percolation, which is done by removing a random sequence of bonds from an initially intact lattice. In this case, bonds are being removed from the lattice with equal probability, i.e., irrespective of the threshold distribution used for fracture.

The macroscopic features of the simulation such as strain and stress are obtained directly from the simulation output, while the statistical analysis of damage clusters requires some postprocessing, mostly taken over from theoretical ${ }^{24,25}$ and numerical ${ }^{26}$ studies of random percolation and extensively described in Ref. 18. The simulations are performed for a sufficiently large set of samples per lattice size (see Table I) in order to obtain statistically reliable data.

In analyzing the damage patterns, we identify clusters of connected broken (removed) bonds and calculate the following microscopic cluster properties as functions of $p$-the density of broken (removed) bonds. The cluster-size distribution $n_{s}$ is the number of clusters of weight $s$ divided by the total number of sites in the lattice. The second moment $M_{2}$ of this distribution plays the role of an important macroscopic measure of the average cluster weight.

The average squared gyration radius $R_{s}$, showing the spatial extent of clusters of a particular weight $s$, is

$$
R_{s}^{2}=\left\langle\sum_{i=1}^{s} \frac{\left|\mathbf{r}_{i}-\mathbf{r}_{\mathrm{c} . \mathrm{m} .}\right|^{2}}{s}\right\rangle_{(\text {(all s-clusters) }},
$$

where $\mathbf{r}_{c . m}$ is the center of mass of an individual cluster and $\mathbf{r}_{i}$ defines the location of each cluster node.

The correlation length $\xi$, which is an average distance between two sites of the same cluster, is

$$
\xi^{2}=\frac{2 \sum_{s} R_{s}^{2} s^{2} n_{s}}{\sum_{s} s^{2} n_{s}} .
$$

In the case of random damage, percolation theory describes the large-scale statistics of the developing cluster distribution and cluster pattern, when local details in the underlying lattice become irrelevant. In particular, it predicts then the following universal scaling laws for an infinite lattice in the vicinity of the percolation threshold $p_{c}$ (Refs. 24 and 25):

$$
\begin{gathered}
M_{2} \propto\left|p-p_{c}\right|^{-\gamma}, \\
\xi \propto\left|p-p_{c}\right|^{-\nu}, \\
n_{s} \propto p s^{-\tau} f\left(s^{3-\tau} / M_{2}\right),
\end{gathered}
$$

where $f$ is a scaling function. The gyration radius $R_{s}$ of a cluster is connected to the cluster size $s$ via an exponent $D_{f}$ known as fractal dimension. The RP exponents take universal values dependent only on dimension $D$ and obeying the following relations in the vicinity of $p_{c}$ :

$$
\frac{D_{f}}{D}=\frac{1}{\tau-1}, \quad \frac{\gamma}{\nu D}=\frac{3-\tau}{\tau-1} .
$$

For $D=2$, one has $\gamma=43 / 18, \nu=4 / 3, D_{f}=91 / 48$, and $\tau$ $=187 / 91$, while $p_{c}$ and all the proportionality factors depend on lattice details. The scaling function $f(z)$ should approach a constant for $z \ll 1$ and decrease to zero for $z \gg 1$.

In order to identify possible anisotropy of the developing fracture pattern, we also calculate longitudinal and transversal correlation lengths $\xi_{\|}$and $\xi_{\perp}$ similar to Eq. (3) but taking into account the distance in one direction instead of the radius. Then, a shape factor can be defined as

$$
\Phi=\frac{\xi_{\perp}-\xi_{\|}}{\xi_{\perp}+\xi_{\|}}
$$

describing the damage anisotropy. For calculating this measure, we always use fixed axes which are linked to the direction of load and then average the axial components of the correlation length separately. Therefore, the shape factor should be equal to zero for random percolation of damage. This is different from the nonzero anisotropy measure found in Ref. 27 for RP, where principle axes of clusters are used.

We define a crack as the biggest cluster at the end of our fracture simulation. The crack profile is derived from the geometry of the biggest cluster in three ways, which will be described later. Once the crack profile is defined, the 


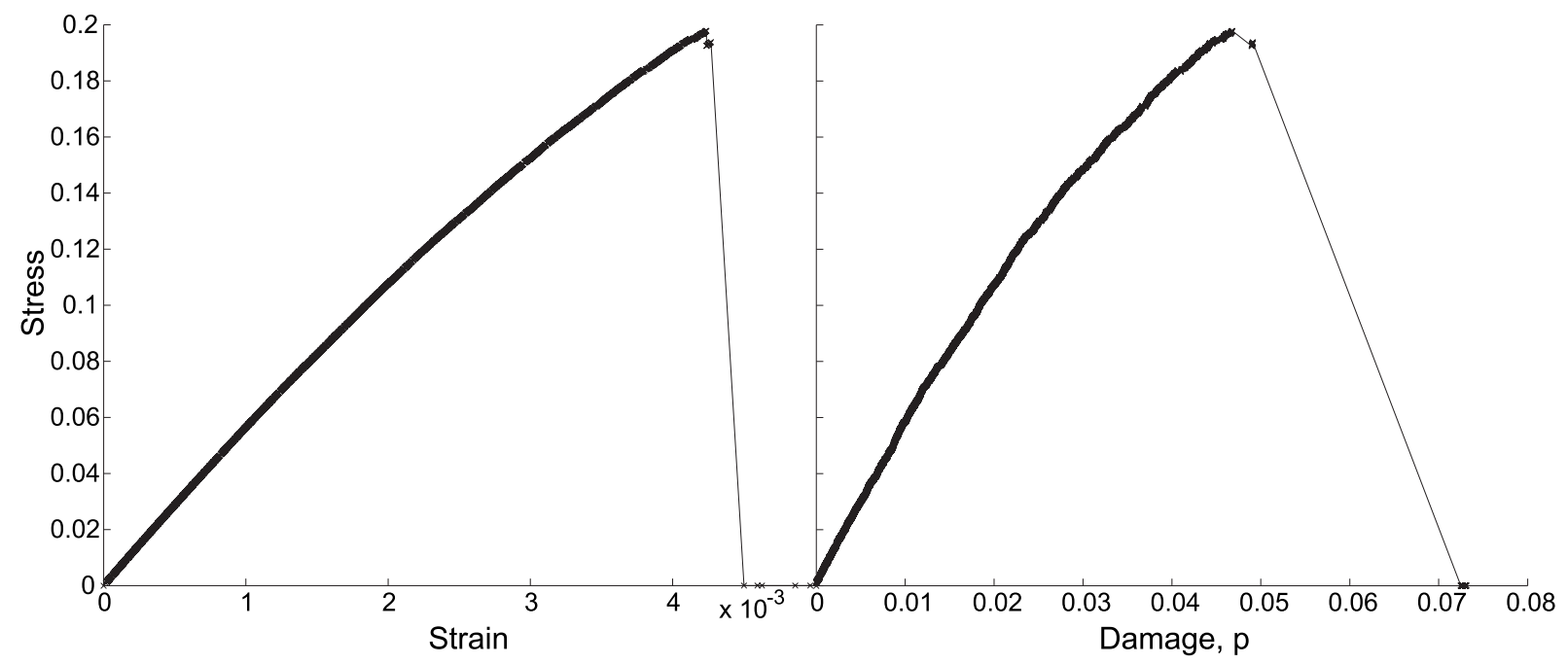

FIG. 2. Stress-strain and stress-damage dependencies for a single fracture simulation with $\alpha=0$ and lattice linear size $L=200$.

roughness $\Delta h$ of the crack can be calculated according to the following formula:

$$
\begin{aligned}
\Delta h= & \left\langle\max \left(h(y), y \in\left[y_{0}, y_{0}+d\right]\right)\right. \\
& \left.-\min \left(h(y), y \in\left[y_{0}, y_{0}+d\right]\right)\right\rangle_{y_{0}},
\end{aligned}
$$

where $y$ is the coordinate along the average crackpropagation direction, $d$ is the width of the averaging window, and $h(y)$ is the height of the crack profile. Note that different methods exist to measure self-affinity exponents. As shown in Ref. 28, these may give different results, in particular, through significant differences in system-size dependence. Also shown in Ref. 28 is that the "variable-bandwidth method using the minimum-maximum difference," which we employ, is by far the most size-insensitive method for selfaffinity exponents around 0.7 and should thus be strongly preferred for the present purpose.
As suggested by experimental data and theoretical predictions, ${ }^{2,3,15,29}$ the scaling relation is expected to be of the form

$$
\Delta h \propto d^{\zeta},
$$

with $\zeta$ denoting the roughness exponent. Arguments of percolation in a gradient ${ }^{15}$ result in a connection between the roughness exponent and RP exponent $\nu$ in the form of $\zeta$ $=\frac{2 \nu}{2 \nu+1}$.

\section{RESULTS}

\section{A. Macroscopic stress and strain}

The stress-strain dependence in our model is typical for heterogeneous materials: bonds start to break from the very beginning and when the critical state is reached, macroscopic failure occurs (Figs 2-4). The critical (maximum) stress and the corresponding critical strain decrease with increasing dis-

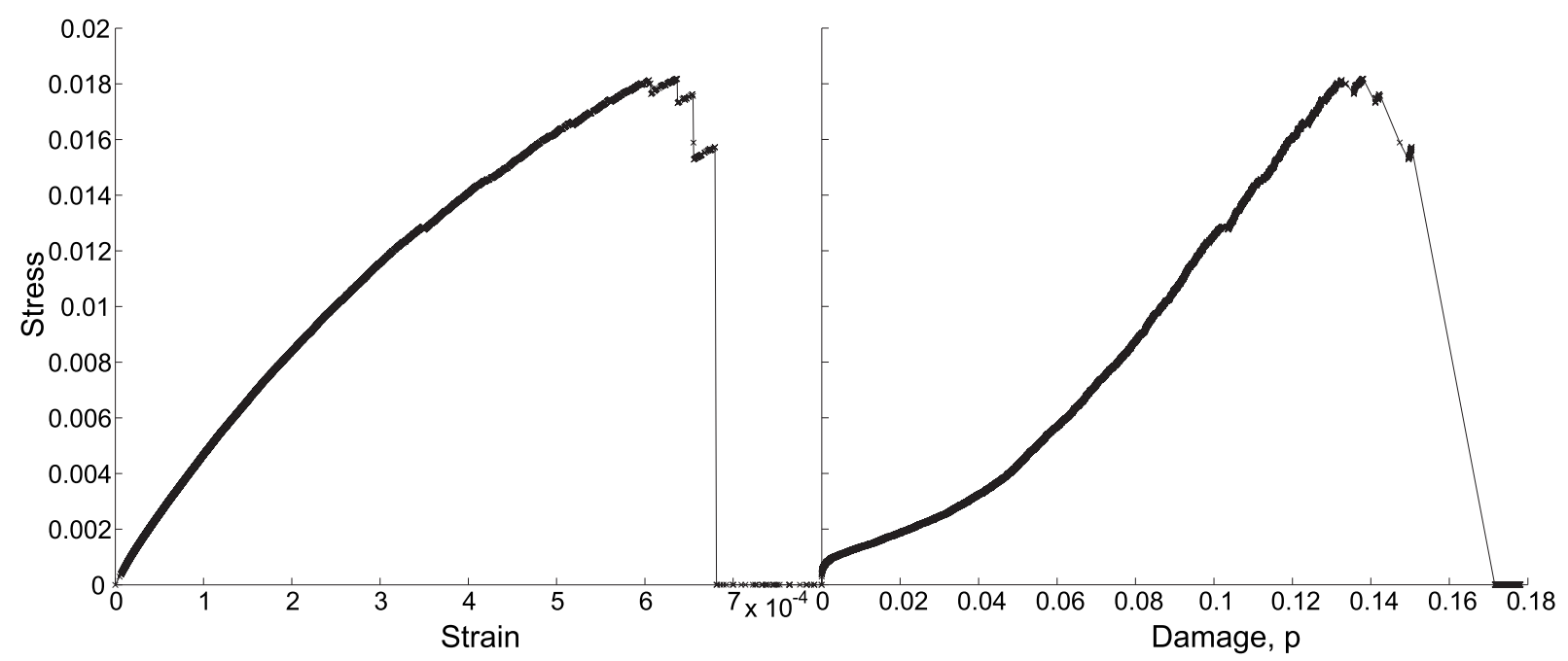

FIG. 3. Stress-strain and stress-damage dependencies for a single fracture simulation with $\alpha=0.6$ and lattice linear size $L=200$. 


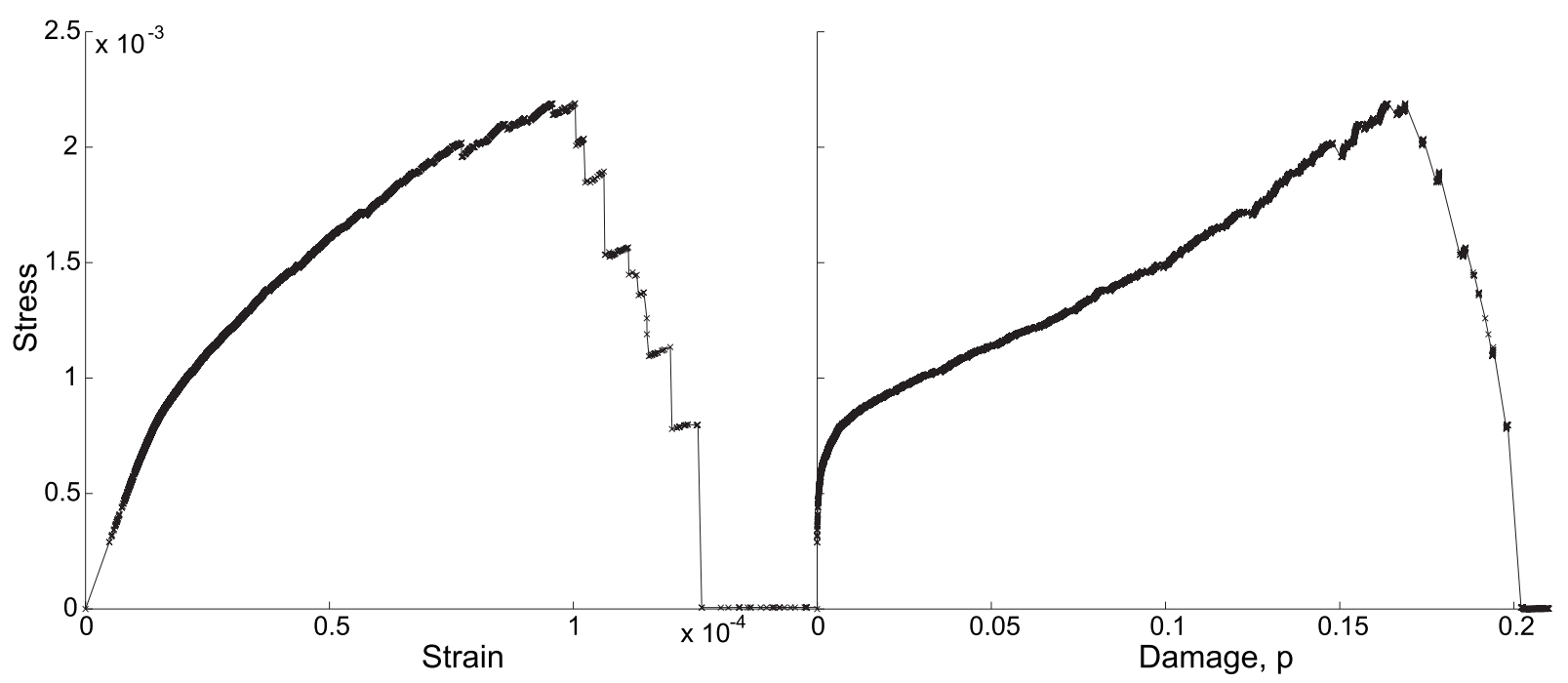

FIG. 4. Stress-strain and stress-damage dependencies for a single fracture simulation with $\alpha=0.75$ and lattice linear size $L=200$.

order strength. This fact is logical since we increase the disorder at a constant value of $w$ in the distribution [Eq. (1)], i.e., we introduce more weak bonds in the lattice.

The shape of the stress-strain curve also changes with $\alpha$. For uniform disorder $(\alpha=0)$, the dependence (both vs strain and vs damage density $p$ ) slightly curves downward until the maximum and then rapidly drops (Fig. 2). For higher disorder (Figs. 3 and 4), the stress-strain curve becomes more convex, while the stress-damage curve gets more concave. The former can be explained by the fact that at constant strain, more bonds are broken for strong disorder than for weak one, which in turn leads to a more significant decrease of the overall stress. The concave shape of the stress-damage curve shows that for larger $p$, breaking becomes more difficult because relatively stronger bonds remain. For strong disorder with $\alpha=0.75$ (Fig. 4), the prefailure ranges of both the stress-strain and stress-damage dependencies are separated into two parts with different curvatures, and the final drop is smoother. It indicates that the prefailure phase consists of two qualitatively different regimes: a very short initial regime where stress builds up quasielastically without much damage, followed by a regime with damage increase and softening like for lower disorder. (In the very early stage, a fully elastic response occurs for $\alpha=0.75$; this is an artifact because when randomly generating thresholds, we suppress thresholds below a fixed level close to zero.) It is seen from Figs. 2-4 that contrary to the critical strain, the critical damage density (density of broken bonds at the point of maximum stress) increases when $\alpha$ becomes larger. This means that for higher disorder, more damage is allowed before failure, which is a consequence of the fact that weak bonds can be relatively harmlessly removed from the lattice without inducing a macroscopic breakdown.

The effect of the disorder strength $\alpha$ on the averaged stress-strain and stress-damage dependencies is presented in Fig. 5. While the initial slope (stiffness) of the stress-strain curve is the same for all values of $\alpha$, the curves representing the dependence vs $p$ change with $\alpha$ from the very beginning, which once again points at the subtle role of disorder in the damage development toward fracture.

We have also studied the size effect in the stress-strain and stress-damage dependencies. As is clear from Fig. 6, such an effect is only present in the region around the maximum-stress point and beyond. The maximum stress and the corresponding critical strain and damage are shifted toward smaller values for bigger lattices. The size dependence of the maximum-stress point can be successfully fitted by a power law $\sigma-\sigma_{\infty} \propto L^{-\kappa}$ (Fig. 7). The corresponding scaling exponent $\kappa$ changes from 0.63 for $\alpha=0$ up to 1.07 for $\alpha$ $=0.75$; so in terms of critical stress, the size effect becomes stronger for stronger disorder. The critical strain can be fitted very well by $\varepsilon-\varepsilon_{\infty} \propto L^{-\lambda}$ (Fig. 8). The exponent $\lambda$ in this case does not change significantly, it slightly increases (from 0.58 to 0.6) with increasing $\alpha$. All numbers together are given in Table II. We warn here that the region around the critical strain is probably a transition between two regimes dominated by different mechanisms, random damage percolation and more avalanchelike behavior. Therefore, not much can be concluded from the scaling in Figs. 7 and 8 until also the postyield regime has been understood in more detail. In fact, the observed scaling of the critical stress and strain may be accidental.

\section{B. Macroscopic features of damage clusters}

Simulation results for the second moment $M_{2}$ of the cluster-size distribution and for the correlation length $\xi$ are given as functions of the damage density $p$ in Figs. 9 and 10, respectively. The second moment shows a gradual trend with decreasing $\alpha$, away from the RP behavior. The correlation length qualitatively deviates from the RP behavior for all $\alpha$ 's, with a marked crossover in the behavior at low damage when going from high disorder $(\alpha=0.75,0.7)$ to lower disorder. We will therefore analyze these two disorder regimes separately and first consider to what extent our previous 


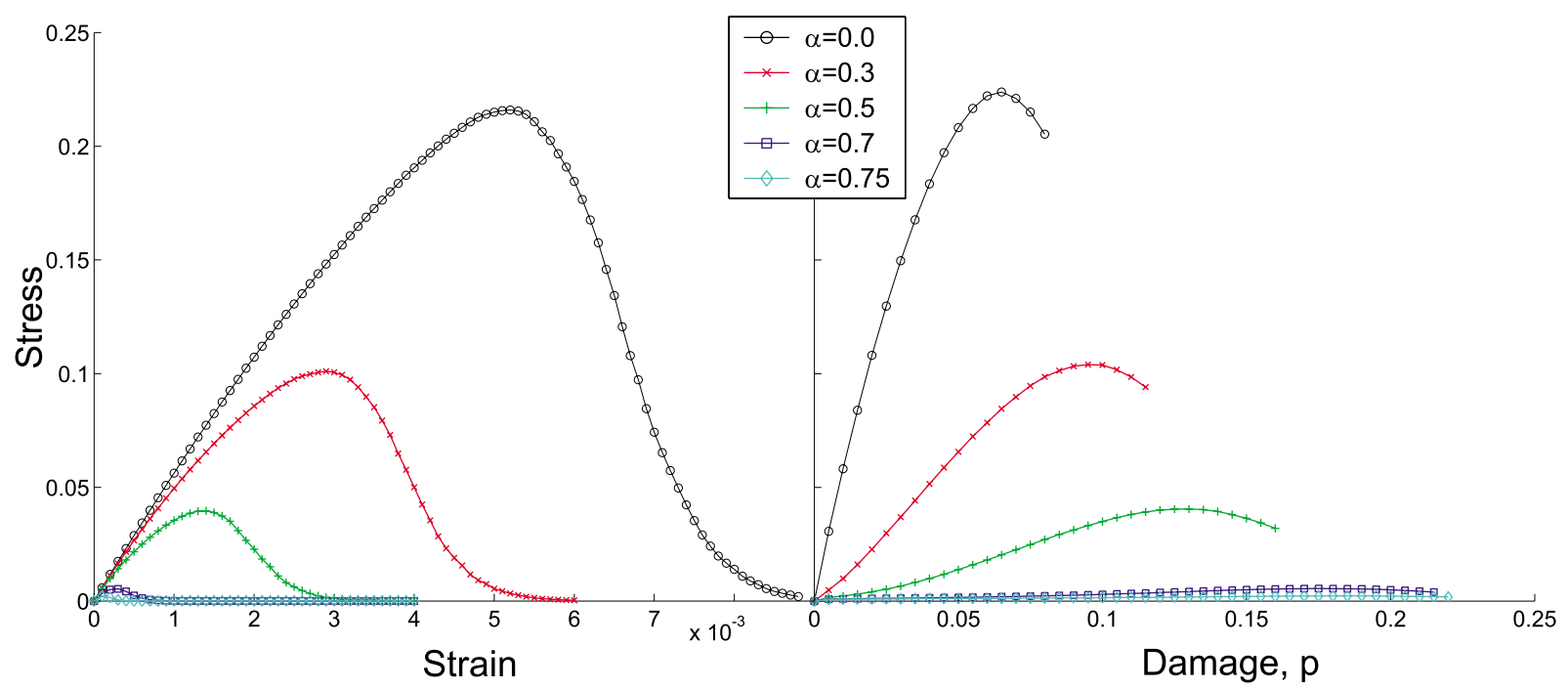

FIG. 5. (Color online) Stress-strain and stress-damage dependencies for different disorder strengths $\alpha$ and for $L=50$; data are averaged over all samples of each particular disorder strength.

conclusions for strong disorder $[\alpha=0.7$ (Ref. 18)] are maintained for $\alpha=0.75$.

\section{High disorder strength}

As was already mentioned, our previous work showed the existence of a percolationlike regime for the case of strong disorder with $\alpha=0.7$. We confirm now the presence of this regime for $\alpha=0.75$ by plotting $M_{2}$ and $\xi$ vs $\left|p-p_{c}\right|$ (Fig. 11). Linear parts of the graphs have slopes matching the ones of percolation theory. As in the case $\alpha=0.7,{ }^{18}$ the percolation thresholds obtained from the two functions $M_{2}(p)$ and $\xi(p)$ are the same and are marginally larger than in that case: $p_{c}$ $=0.28(\alpha=0.75)$ vs $p_{c}=0.27 \quad(\alpha=0.7)$. The existence of a percolationlike regime can be shown without assuming a certain value of $p_{c}$, by plotting $M_{2}^{-18 / 43}$ versus $\xi^{-3 / 4}$ and checking the possibility to fit the dependency by a straight line through the origin. The result (Fig. 12) is fully consistent with Fig. 11, where we try to match the proper slopes. Similarity with random percolation is also confirmed by scaling of the cluster-size distribution [Eq. (6) and Figs. 13 and 14]: for the theoretical RP Fisher exponent $(\tau=187 / 91)$, the data allow the construction of a scaling function with a plateau at low arguments.

Attempts to build a scaling function for fracture with $\alpha$ $=0.7$ and $\alpha=0.75$ by using $\tau$ different from the theoretical value of RP do not lead to satisfactory results (not shown). A scaling function still seems to be plausible for $\tau 20 \%$ lower than the theoretical value but this function does not have a plateau at low arguments. For $\tau 20 \%$ higher than $187 / 91$, the

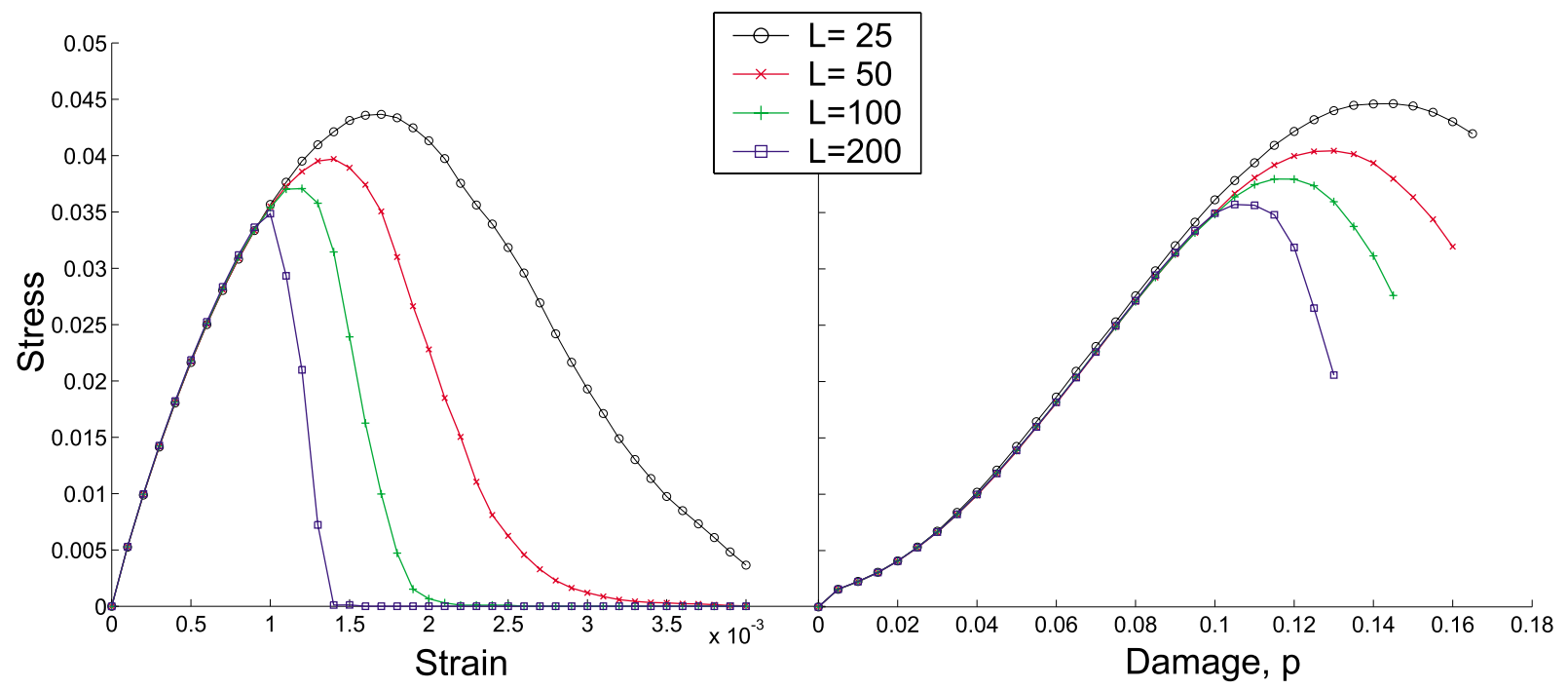

FIG. 6. (Color online) Stress-strain and stress-damage dependencies for different lattice sizes $L$ and for $\alpha=0.5$; data are averaged over all samples of each particular lattice size. 


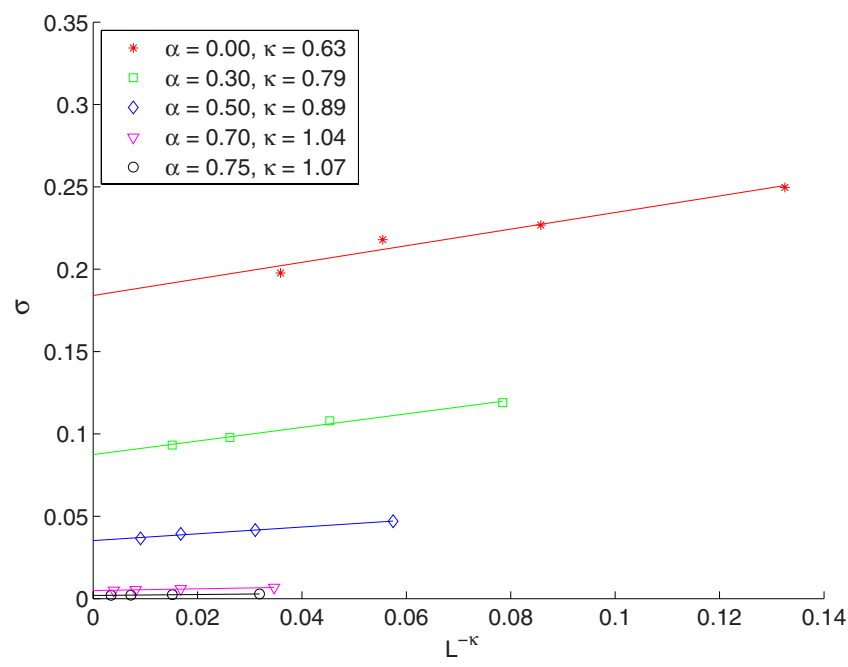

FIG. 7. (Color online) Scaling of the critical stress, least-squares fitting.

attempt to build the scaling function is unsuccessful.

Analogous to Eq. (6), the scaling relation for $n_{s}$ can be based on $\xi$ instead of $M_{2}$ and written in a form consistent with $\nu=4 / 3$,

$$
n_{s} s^{\tau} \propto g\left(s^{2 / D_{f}} \xi^{2}\right)
$$

Again, the scaling function should have a plateau at low arguments. The result is very similar to Fig. 14, though a small deviation starts to be noticeable at the highest concentration of the assumed scaling regime, $p=0.18$. An attempt to use $D_{f} 20 \%$ higher or lower than its theoretical value for random percolation (not shown) leads to the worse quality of the result. We test the appropriateness of the theoretical RP value of $D_{f}$ for our fracture data in an independent way, as

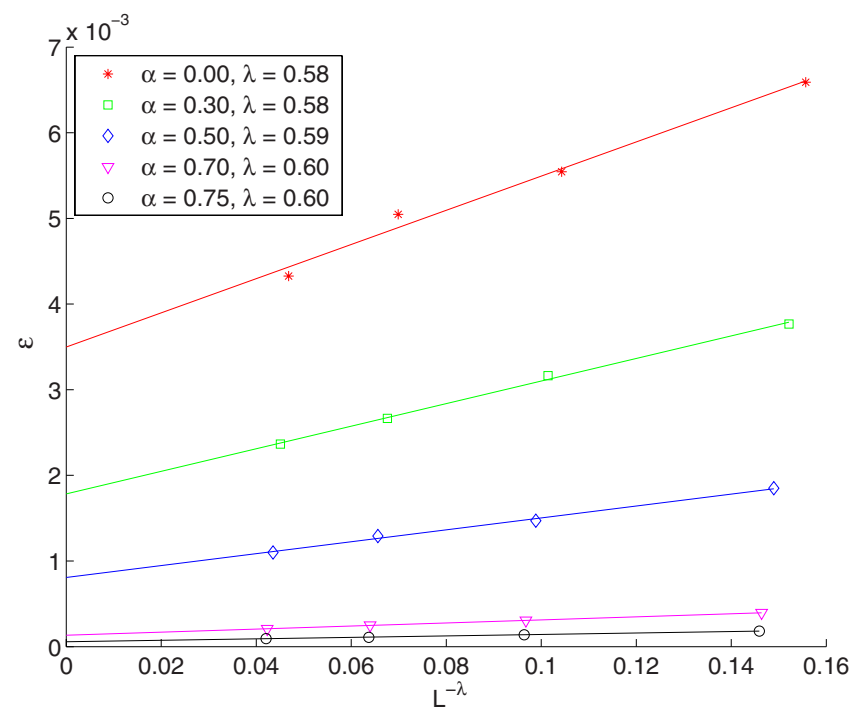

FIG. 8. (Color online) Scaling of the critical strain, least-squares fitting.

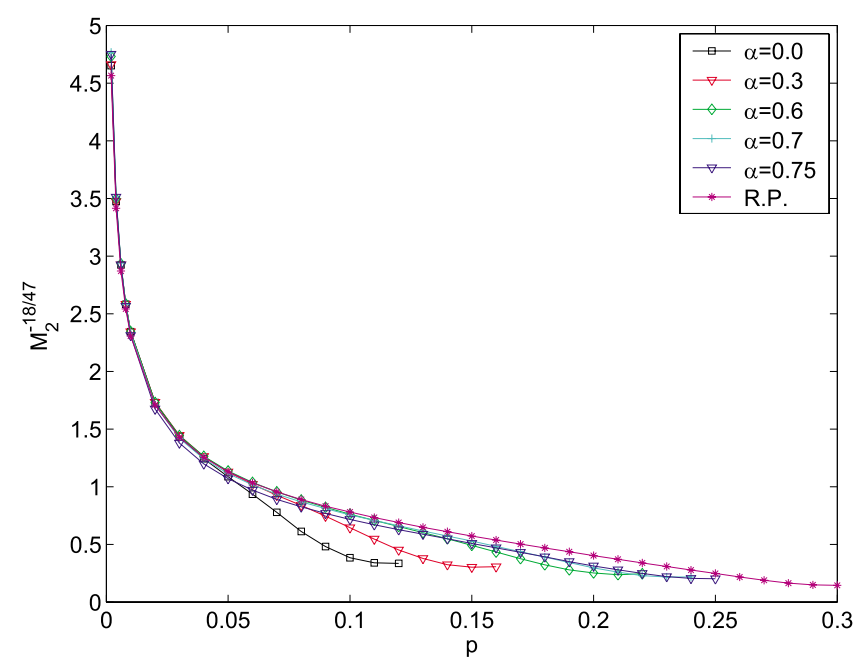

FIG. 9. (Color online) Second moment $M_{2}$ of the cluster-size distribution for lattice linear size $L=50$; data represent fracture simulations with different values of $\alpha$ and the simulation of random percolation.

the scaling exponent of the gyration radius of damage clusters $R_{g}(s)$. Fitting of the exponent gives $D_{f}=1.894$ (not shown), which is closely consistent with the theoretical value $D_{f}=91 / 48 \approx 1.896$.

So we conclude that indeed the data for damage development in fracture at high disorder are consistent with RP and with the theoretical RP exponents, but in a limited damageconcentration regime only. The percolationlike regime is slightly wider for $\alpha=0.75$ than for $\alpha=0.7,{ }^{18}$ but it gets significantly smaller for $\alpha=0.65$ and almost disappears for $\alpha$ $=0.6$ (not shown). The regime is not observed at all for lower values of the disorder strength.

For low damage density $p$, a qualitative deviation of the cluster correlation length $\xi$ from the random-percolation behavior is observed. The deviation is more pronounced in the case of the highest values of $\alpha$ (Fig. 10). Contrary to that, the second moment of the cluster-size distribution is quite close to the random-percolation data in the range of low $p$. A localization process with a characteristic length scale comparable to a bond length can be the cause for the deviation: ${ }^{18}$ if the disorder is strong, the probability of two very weak bonds to be close to each other is high. This, in turn, makes these neighboring weak bonds break next to each other, causing short-range localization.

TABLE II. Scaling exponents for critical stress and strain.

\begin{tabular}{lcc}
\hline \hline$\alpha$ & $\begin{array}{c}\text { Critical-strain } \\
\text { exponent } \lambda\end{array}$ & $\begin{array}{c}\text { Critical-stress } \\
\text { exponent } \kappa\end{array}$ \\
\hline 0 & 0.58 & 0.63 \\
0.3 & 0.58 & 0.79 \\
0.5 & 0.59 & 0.89 \\
0.7 & 0.60 & 1.04 \\
0.75 & 0.60 & 1.07 \\
\hline \hline
\end{tabular}




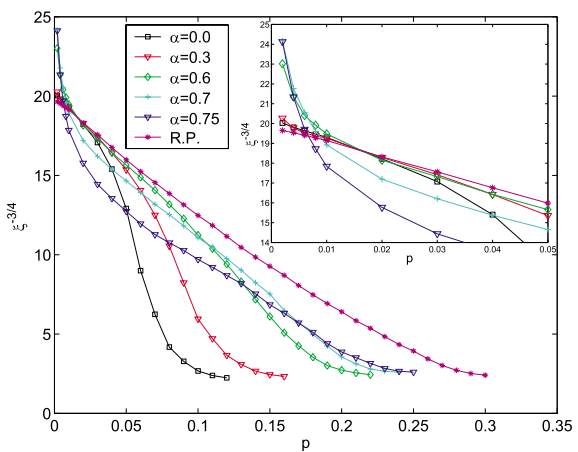

FIG. 10. (Color online) Correlation length $\xi$ of damage clusters for lattice linear size $L=50$; data represent fracture simulations with different values of $\alpha$ and the simulation of random percolation.

\section{Low disorder strength}

The gradual change with $\alpha$ of the second moment $M_{2}$ certainly does not disprove the presence of a percolationlike regime for weak disorder; on the basis of this graph, one still can think that there is a short linear part of the graph corresponding to scaling. Qualitative changes in $\xi$ suggest, how-

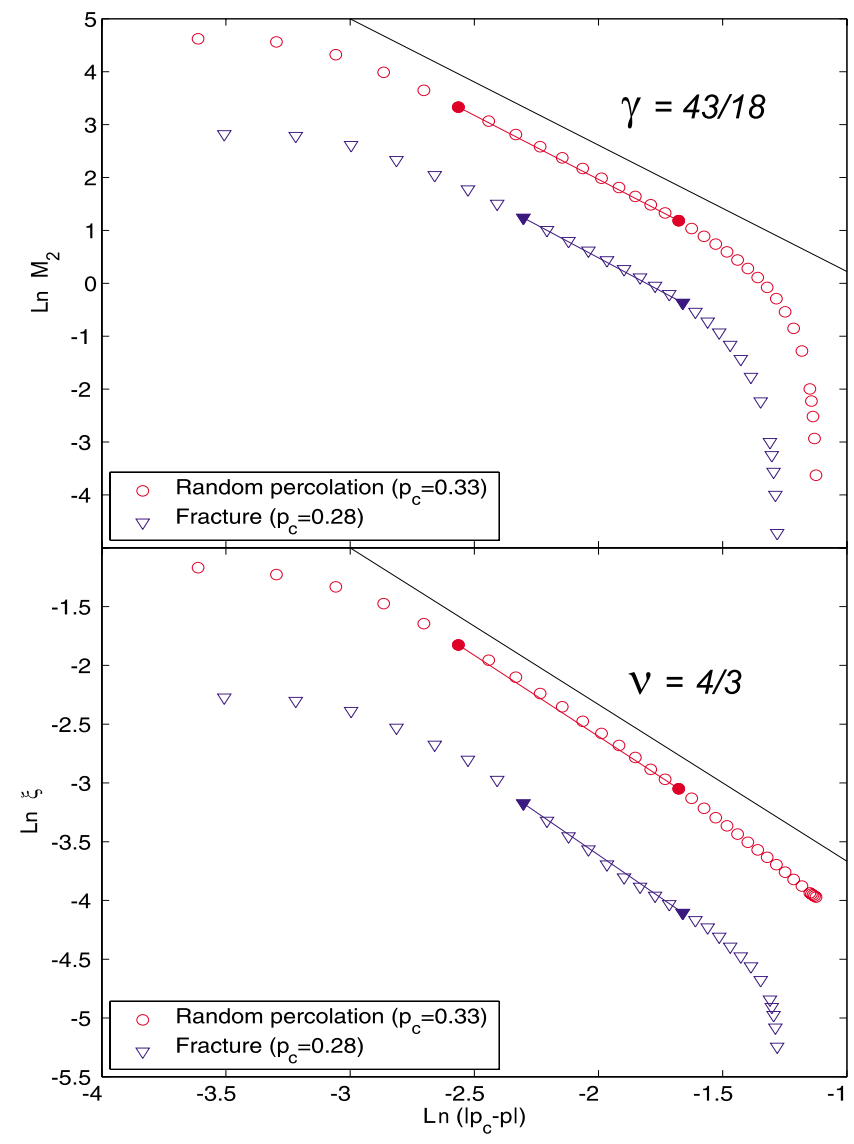

FIG. 11. (Color online) Scaling of the second moment $M_{2}$ of the cluster-size distribution and of the correlation length $\xi$ as functions of $\left|p_{c}-p\right|$ for simulations with linear lattice size $L=50$ and for $\alpha$ $=0.75$. Limits of the scaling regime are denoted by solid markers; the black solid lines show the theoretical RP slopes; curves are shifted from each other for clarity.

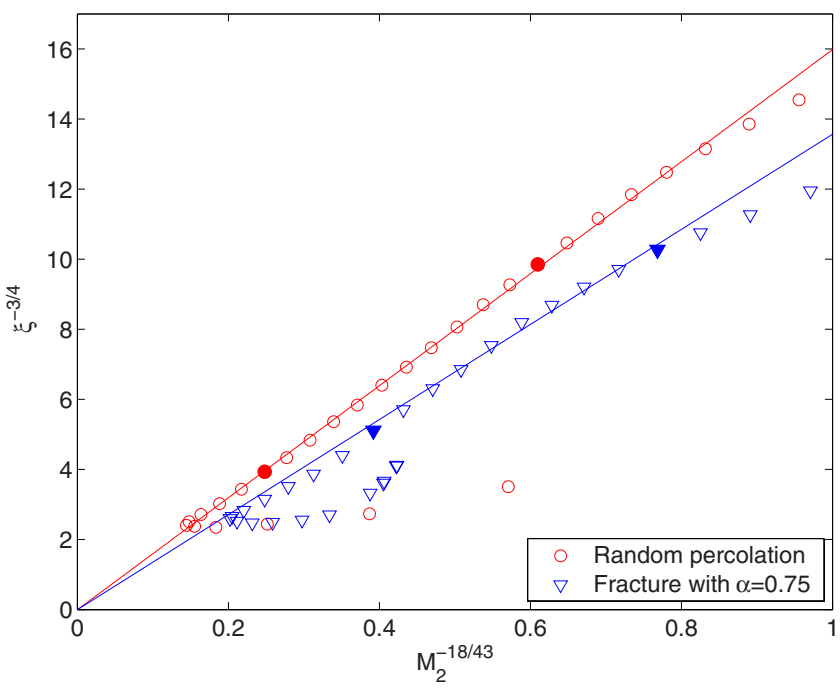

FIG. 12. (Color online) Second moment $M_{2}$ of the cluster-size distribution versus correlation length $\xi$, both scaled with proper RP exponents, for simulations with linear lattice size $L=50$ and for $\alpha$ $=0.75$. Limits of the scaling regime are denoted by solid markers; the solid lines represent least-squares fits through the origin.

ever, that different processes are going on in the cases of strong and weak disorders. This can be made clear by considering the anisotropy factor $\Phi$ [Eq. (8)]. It is plotted in Fig. 15 for different values of $\alpha$. The anisotropy factor is obviously zero for RP. For fracture, $\Phi$ has a plateau close to zero in the region of isotropic damage development. The plateau and, correspondingly, the region of possible similarity between the fracture and RP are only present in fracture simulations with $\alpha \geqslant 0.6$. An attempt to scale the graphs of the anisotropy factor with the damage density at the maximum stress $p_{\text {max stress }}$ seems feasible again only for $\alpha \geqslant 0.6$. For lower values of $\alpha$, there is apparently no similarity between the fracture and random percolation: the range of damage density with an isotropic cluster development is absent and,

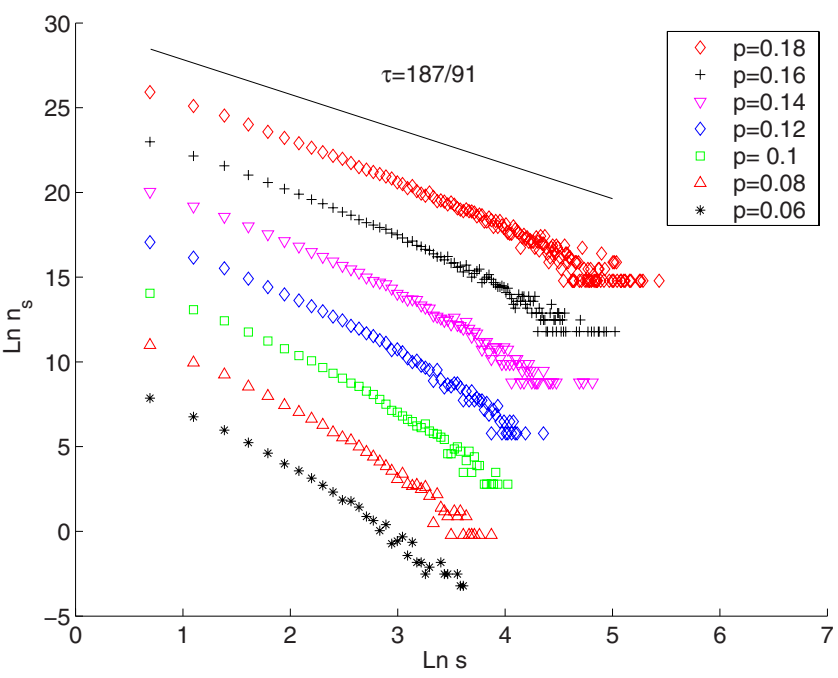

FIG. 13. (Color online) Evolution of the cluster-size distribution: $\alpha=0.75, L=50$. 


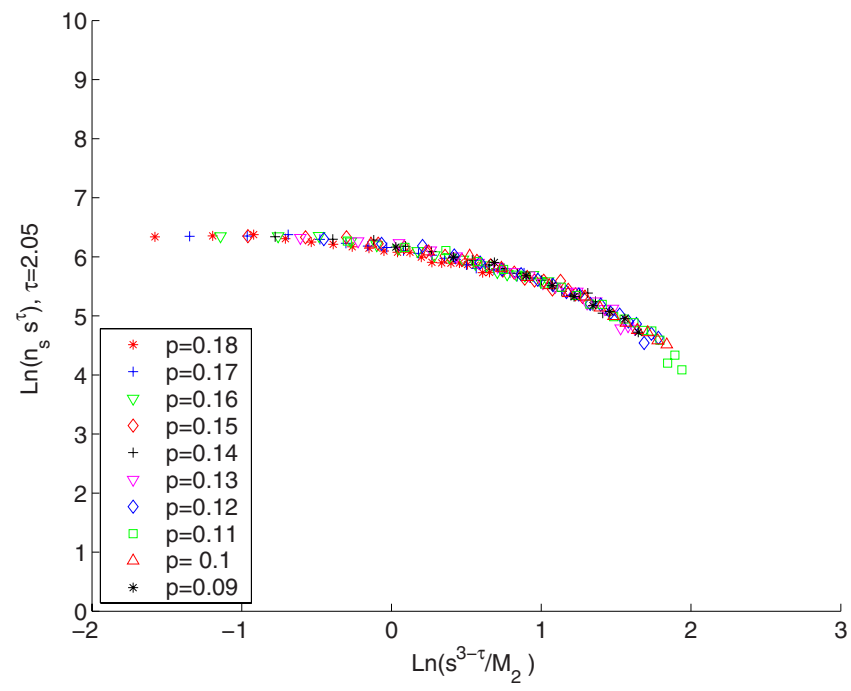

FIG. 14. (Color online) Scaling function of the cluster-size distribution for fracture simulation within the scaling regime ( $p$ $=0.09-0.18 ; \alpha=0.75)$. The data represent averages over all samples of size $L=50$; scattered data for large $s$ are excluded; curves are leveled vertically to match one another [cf. Eq. (6)].

as stated above, we observed that a scaling regime like the one present in Fig. 11 vanishes below $\alpha=0.6$.

For low $\alpha$, the short-range localization at low $p$ is not observed because weak bonds are not likely located close. However, there is another feature typical for low disorder but not observed for high $\alpha$ : the anisotropy factor has negative values during the very early deformation stage. This suggests that in the beginning of the fracture process, mostly isolated single bonds oriented parallel to the load are being broken. This fact is rather logical: since the difference in thresholds is not huge, orientation influences the choice of a bond to be broken much more than it does for high disorder strength.

In summary, only for $\alpha=0.6$ and higher there is a regime in which damage develops in an isotropic way and in which both $M_{2}$ and $\xi$ follow scaling laws consistent with random percolation of damage and with the theoretical RP exponents $\gamma=43 / 18, \nu=4 / 3$.

\section{E. Localization and anisotropy}

Both for high and low $\alpha$, the regime of damage development with low to moderate anisotropy is limited from above by the onset of strong anisotropy in the damage clusters, which signals the macroscopic localization of damage (Fig. 15). It is clearly seen that the curve corresponding to the highest $\alpha$ shows a noticeable increase of anisotropy at $p$ $=0.18$. This is most likely the cause of the small deviation of the scaling function $g\left(s^{\tau-1} / \xi^{2}\right)$ at $p=0.18$ that was mentioned above. As seen from the damage-development snapshots (Figs. 16 and 17) and damage-density profiles (Fig. 18) for the largest available lattice size $L=200$, the macroscopic localization comes into play at (or very close to) the point of maximum stress both for uniform $(\alpha=0)$ and high $(\alpha$ $=0.75$ ) disorders. If one looks only at the damage snapshots and the damage-density profiles, it might be tempting to conclude that for high, moderate, or uniform disorder, the picture remains qualitatively the same: visually, damage appears to develop in a percolationlike way up to the maximumstress point. However, as made clear above, our analysis of the scaling laws and anisotropy factor show a clear difference in the damage evolution for lattices with low ( $\alpha$ $<0.6)$ and high $(\alpha \geqslant 0.6)$ disorder strengths.

Thus, macroscopic localization limits the percolationlike regime in the case of strong disorder from above. For low disorder, it takes over from the initial regime without any percolationlike intermediate phase. A question remains to which extent the distinction in disorder is size dependent, but here computing resources remain limited.

\section{F. Crack roughness}

We will analyze the crack-roughness scaling as defined in Eqs. (9) and (10) and investigate the trend with disorder

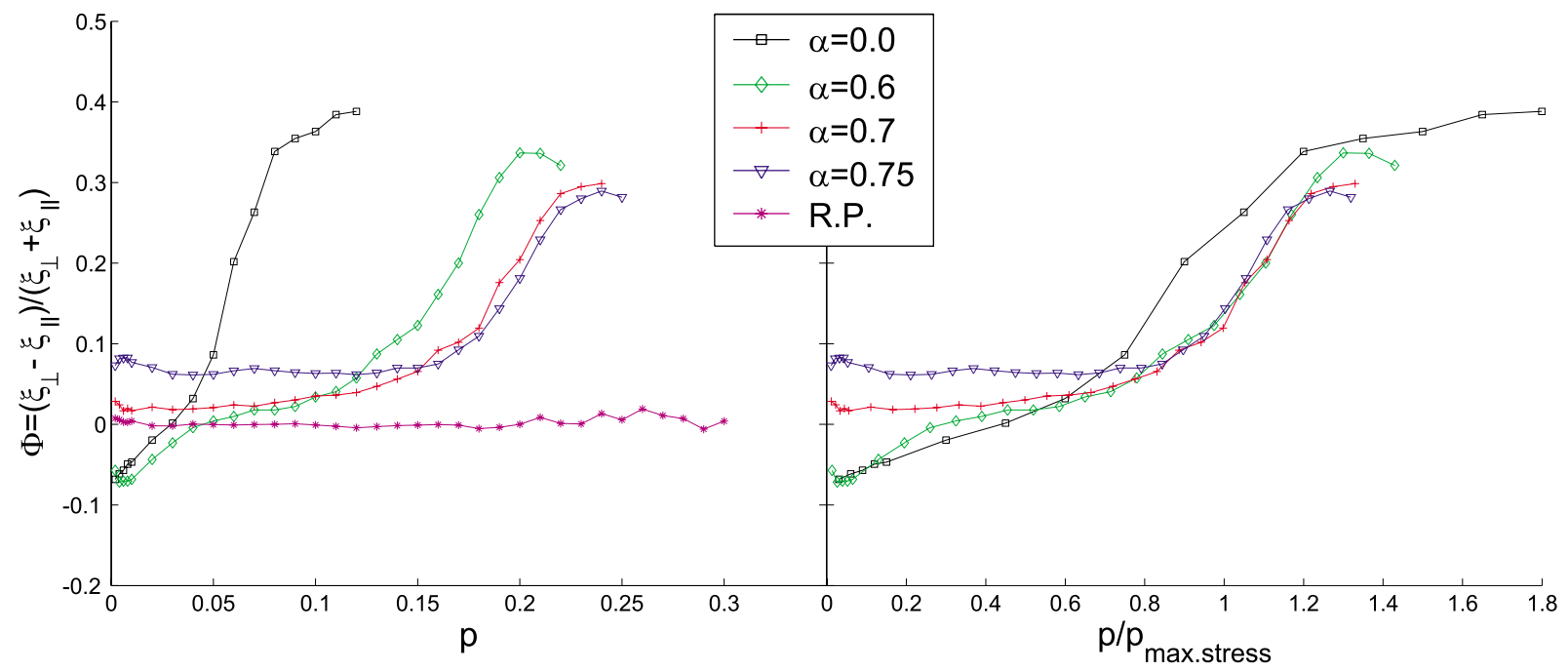

FIG. 15. (Color online) Anisotropy factor $\Phi$ of damage clusters for lattice linear size $L=50$; data represent fracture simulations with different values of $\alpha$ and the simulation of random percolation. 

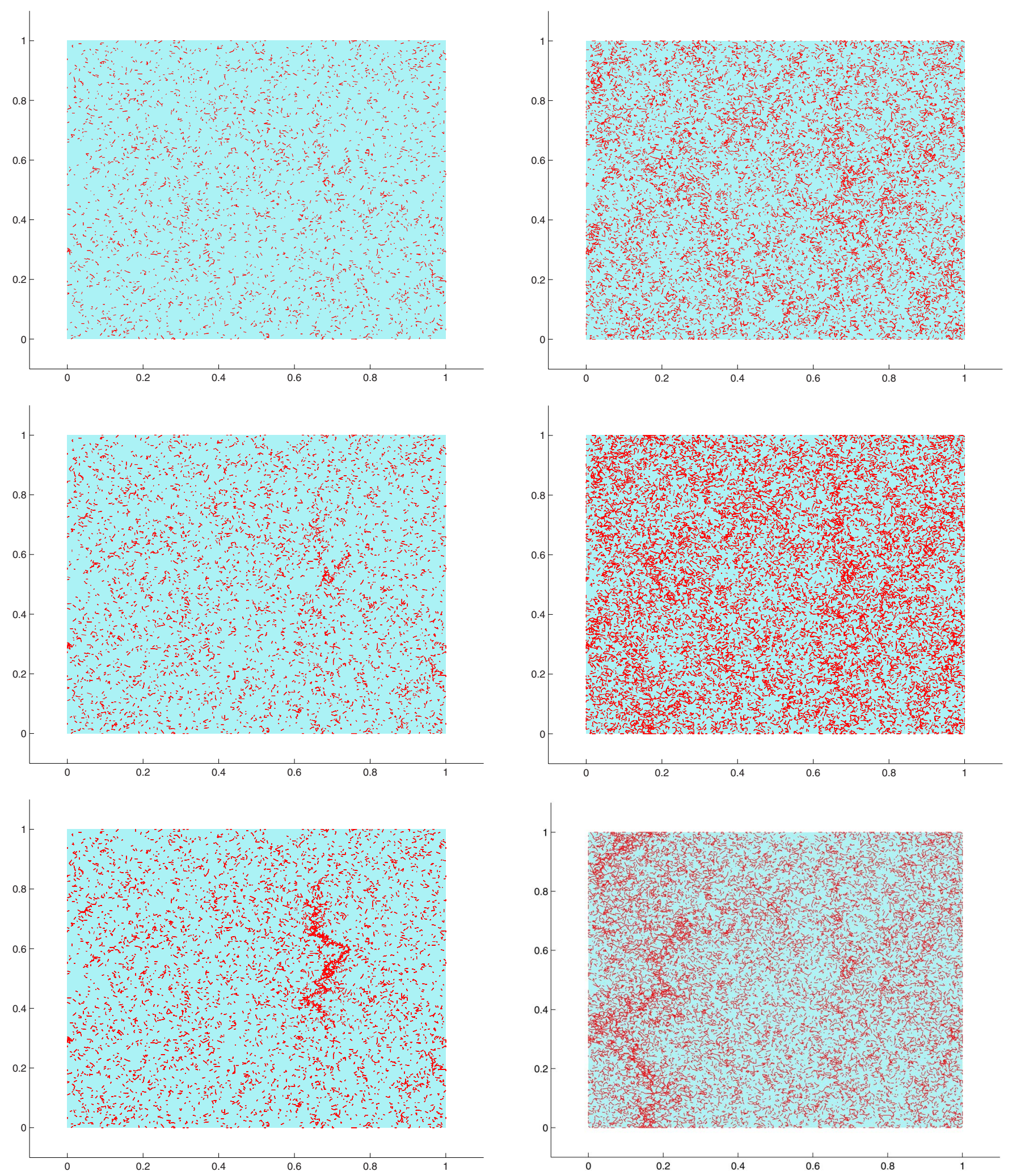

FIG. 16. (Color online) Snapshots for a single fracture simulation with $\alpha=0$ and with lattice linear size $L=200$.

strength. We employ only the simulations with $L \geqslant 100$ in order to avoid the effect of boundaries. As explained in our previous paper, ${ }^{18}$ the crack path can be defined in at least three ways: averaging, in the direction of load, of the biggest

FIG. 17. (Color online) Snapshots for a single fracture simulation with $\alpha=0.75$ and with lattice linear size $L=200$.

cluster, or identifying the backbone of the biggest cluster by means of the "burning" algorithm, ${ }^{30}$ or using the so-called "solid-on-solid approximation." ${ }^{31}$ In the latter method, one defines the border of intact material touching the biggest 

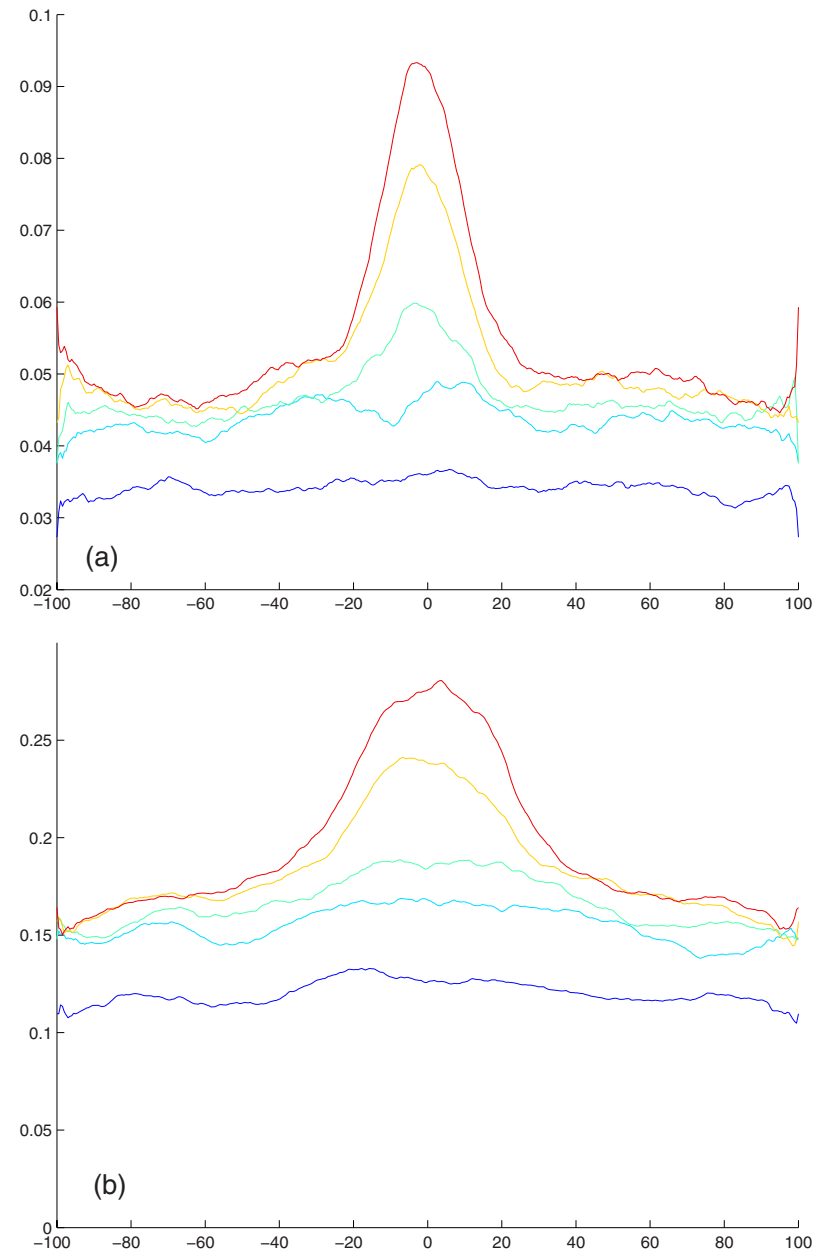

FIG. 18. (Color online) Centered averaged damage-density profiles for simulations with (a) $\alpha=0$ and (b) $\alpha=0.75$ and with lattice linear size $L=200$; curves represent (from bottom upward) damage densities $p_{1}=0.7 p_{\text {max stress }}, p_{2}=0.95 p_{\text {max stress }}, p_{3}=1.0 p_{\text {max stress }}, p_{4}$ $=1.1 p_{\text {max }}$ stress,$p_{5}=1.2 p_{\text {max stress }}$.

cluster from each side. To avoid artifacts, we consider these methods only above length scales larger than the typical damage width. ${ }^{18}$ After the crack-path definition, we estimate the roughness of the obtained curve and its scaling exponent by least-squares fitting of Eq. (10) with Eq. (9). The three methods give different profiles and, consequently, different roughness exponents (Fig. 19). In Ref. 18, we concluded for $\alpha=0.7$ that the two values of $\zeta$ (corresponding to the averaging method and to the burning algorithm) were within errors sufficiently close to be still considered the same. However, we now recognize from Table III that the difference is systematic, so the definition of crack path is important. However, more relevant to the subject of the present paper, we notice that in either definition, the roughness is independent of the disorder strength and hence independent of the damage-development scenario up to the maximum stress. As noted already in Ref. 18, the values of $\zeta$ are close to those observed experimentally for quasi-two-dimensional cracks in wood $(0.68 \pm 0.04) .^{3}$ The value of $\zeta$ based on the backbone roughness almost coincides with that following from random percolation in a gradient $(\zeta=8 / 11=0.73) .{ }^{15}$ Our results for $\zeta$ corresponding to the burning method agree very well with
TABLE III. Roughness exponents obtained from the fracture simulations. (Note: here, the calculation of the roughness exponent for the case of $\alpha=0.7$ is performed for a larger set of samples than in Ref. 18, which leads to somewhat different numbers.)

\begin{tabular}{lccc}
\hline \hline & \multicolumn{3}{c}{ Roughness exponent } \\
\cline { 2 - 4 }$\alpha$ & (Averaging) & ("Burning") & ("Solid on solid") \\
\hline 0.0 & $0.68 \pm 0.10$ & $0.77 \pm 0.10$ & $0.63 \pm 0.09$ \\
0.3 & $0.66 \pm 0.11$ & $0.74 \pm 0.11$ & $0.63 \pm 0.09$ \\
0.5 & $0.66 \pm 0.11$ & $0.74 \pm 0.11$ & $0.62 \pm 0.11$ \\
0.7 & $0.65 \pm 0.11$ & $0.75 \pm 0.11$ & $0.63 \pm 0.10$ \\
0.75 & $0.64 \pm 0.10$ & $0.76 \pm 0.12$ & $0.62 \pm 0.11$ \\
\hline \hline
\end{tabular}

the results of another recent simulation of central-force lattices $\quad(\zeta=0.75 \pm 0.03)$, with even stronger disorder $(\alpha=0.95),{ }^{31}$ although there a different assumption has been made about the scaling of the correlation length.

\section{CONCLUSIONS}

In the present paper, we have numerically studied the fracturing of 2D central-force networks with power-law disorder systematically as a function of the disorder strength. In particular, we have analyzed the macroscopic stress-strain behavior and microscopic damage development and compared the microscopic statistics of the damage clusters with that for random damage percolation. The major conclusion from this work is that the pattern of damage development is qualitatively different for high and low disorders. This sheds new light on conclusions from similar studies in the literature which use a variety of disorder strengths. ${ }^{13-18}$ We have also analyzed the dependence of our results on system size, but rigorous conclusions in this respect will require significantly more computer power than is currently available to us.

For high disorder, i.e., a high power-law exponent $\alpha$ $=0.75$, we confirm our earlier findings [for $\alpha=0.7$ (Ref. 18)] that damage in fracture develops in three stages: short-range localization at low damage concentration $p$, damage development in a middle range consistent with random percolation, and macroscopic damage localization toward a final anisotropic crack. The percolationlike regime rapidly shrinks with decreasing disorder exponent and vanishes around $\alpha$ $=0.6$.

For still lower values of $\alpha$, the damage development is anisotropic from the beginning, with a $90^{\circ}$ change in anisotropy with the increase of the damage; the short-range localization at low $p$ is no longer observed. These results can be rationalized from the disorder statistics.

Both for low and high disorders, macroscopic localization sets in around the maximum stress. While in the damage growth toward the final fracture clear differences are visible between high and low disorders, the roughness of the crack "surface" is surprisingly independent of $\alpha$ and can be characterized by an accurate size-scaling law. The precise value of the roughness-scaling exponent depends on details of the definition of the crack surface but is anyway in good agree- 

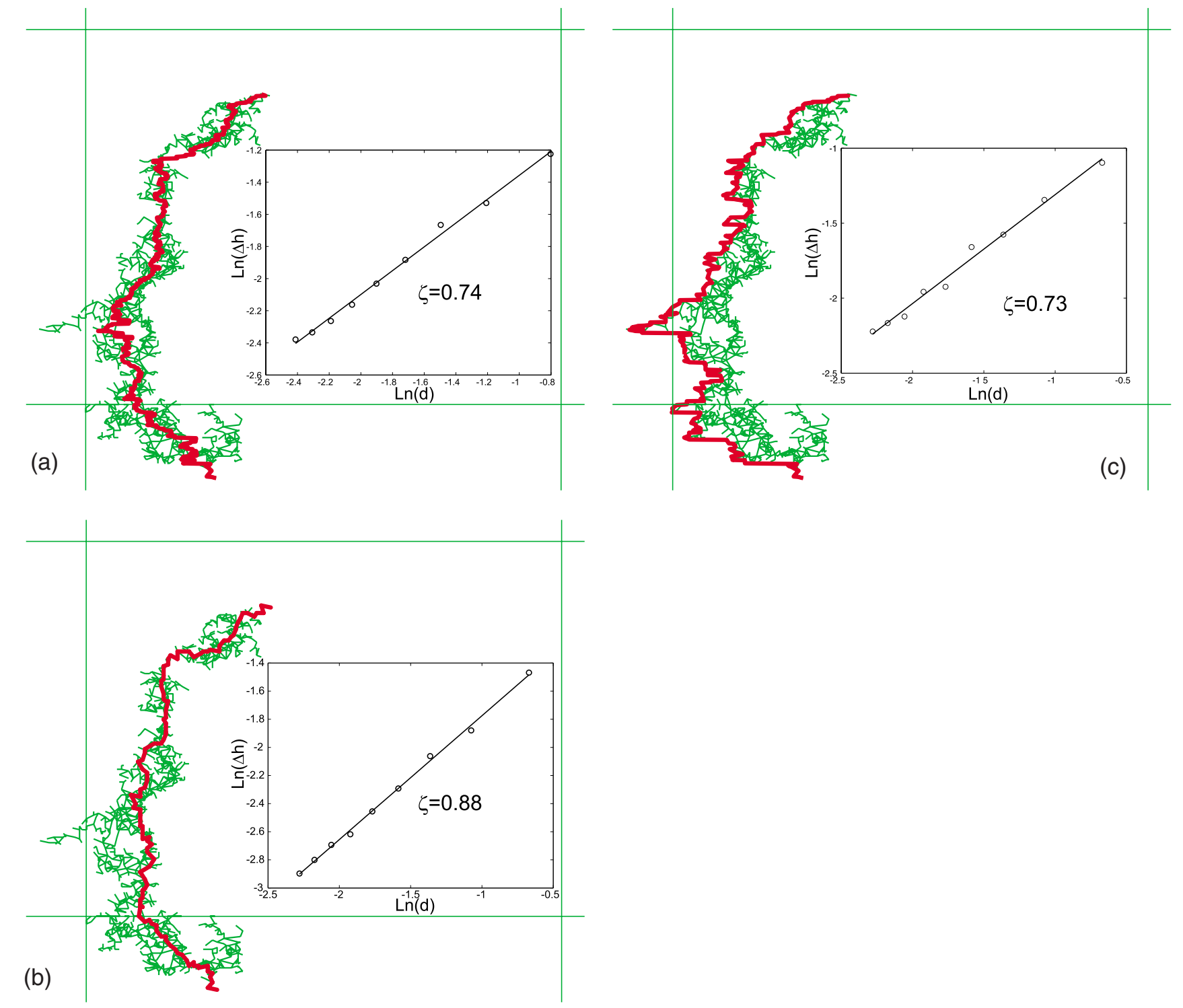

FIG. 19. (Color online) Example of the final crack profile [red (dark gray) line] obtained (a) by means of horizontal averaging, (b) by the burning algorithm, and (c) by the solid-on-solid approximation, for a single simulation with $L=100$; insets represent the scaling of crack roughness for the corresponding profiles.

ment with other numerical and experimental results.

\section{ACKNOWLEDGMENTS}

This work is a part of the research programme of the
Stichting voor Fundamenteel Onderzoek der Materie (FOM), which is financially supported by the Nederlandse Organisatie voor Wetenschappelijk Onderzoek (NWO). The computer facilities for this research were provided by Stichting Nationale Computerfaciliteiten (NCF). *i.malakhovsky@tue.nl

${ }^{1}$ Z. P. Bažant, Arch. Appl. Mech. 69, 703 (1999).

${ }^{2}$ S. Morel, E. Bouchaud, J. Schmittbuhl, and G. Valentin, Int. J.

Fract. 114, 307 (2002).

${ }^{3}$ T. Engøy, K. J. Måløy, A. Hansen, and S. Roux, Phys. Rev. Lett. 73, 834 (1994).

${ }^{4}$ K. J. Maloy, A. Hansen, E. L. Hinrichsen, and S. Roux, Phys.
Rev. Lett. 68, 213 (1992).

${ }^{5}$ K. Takahashi, M. Kido, and K. Arakawa, Int. J. Fract. 90, 119 (1998).

${ }^{6}$ F. Lapique, P. Meakin, J. Feder, and T. Jossang, J. Appl. Polym. Sci. 86, 973 (2002).

${ }^{7}$ S. Morel, E. Bouchaud, and G. Valentin, Phys. Rev. B 65, 104101 (2002). 
${ }^{8}$ E. Bouchaud, J. P. Bouchaud, D. S. Fisher, S. Ramanathan, and J. R. Rice, J. Mech. Phys. Solids 50, 1703 (2002).

${ }^{9}$ A. Parisi, G. Caldarelli, and L. Pietronero, Europhys. Lett. 52, 304 (2000).

${ }^{10}$ E. T. Seppälä, V. I. Räisänen, and M. J. Alava, Phys. Rev. E 61, 6312 (2000).

${ }^{11}$ C. Moukarzel and P. M. Duxbury, Phys. Rev. E 59, 2614 (1999).

${ }^{12}$ S. Roux, A. Hansen, H. J. Herrmann, and E. Guyon, J. Stat. Phys. 52, 237 (1988).

${ }^{13}$ H. J. Herrmann, A. Hansen, and S. Roux, Phys. Rev. B 39, 637 (1989).

${ }^{14}$ M. Sahimi and S. Arbabi, Phys. Rev. B 47, 713 (1993).

${ }^{15}$ A. Hansen and J. Schmittbuhl, Phys. Rev. Lett. 90, 045504 (2003).

${ }^{16}$ P. K. V. V. Nukala, S. Šimunović, and S. Zapperi, J. Stat. Mech.: Theory Exp. P08001, 1 (2004).

${ }^{17}$ Phani Kumar V. V. Nukala, S. Zapperi, and S. Šimunović, Phys. Rev. E 71, 066106 (2005).

${ }^{18}$ I. Malakhovsky and M. A. J. Michels, Phys. Rev. B 74, 014206 (2006).

${ }^{19}$ M. Sahimi, Heterogeneous Materials I. Linear Transport and Optical Properties (Springer-Verlag, New York, 2003).
${ }^{20}$ M. Sahimi, Heterogeneous Materials II. Nonlinear and Breakdown Properties and Atomistic Modelling (Springer-Verlag, New York, 2003).

${ }^{21}$ A. Hansen, E. L. Hinrichsen, and S. Roux, Phys. Rev. B 43, 665 (1991).

${ }^{22}$ S. Arbabi and M. Sahimi, Phys. Rev. B 47, 695 (1993).

${ }^{23}$ R. Riedinger, M. Habar, P. Oelhafen, and H. J. Güntherodt, J. Comput. Phys. 74, 61 (1988).

${ }^{24}$ D. Stauffer and A. Aharony, Introduction to Percolation Theory, 2nd ed. (Taylor \& Francis, London, 1992).

${ }^{25}$ G. Grimmett, Percolation (Springer-Verlag, New York, 1989).

${ }^{26}$ H.-P. Hsu and M.-C. Huang, Phys. Rev. E 60, 6361 (1999).

${ }^{27}$ F. Family, T. Vicsek, and P. Meakin, Phys. Rev. Lett. 55, 641 (1985).

${ }^{28}$ J. Schmittbuhl, J. P. Vilotte, and S. Roux, Phys. Rev. E 51, 131 (1995).

${ }^{29}$ A. Omeltchenko, J. Yu, R. K. Kalia, and P. Vashishta, Phys. Rev. Lett. 78, 2148 (1997).

${ }^{30}$ H. J. Herrmann, D. C. Hong, and H. E. Stanley, J. Phys. A 17, L261 (1984).

${ }^{31}$ J. Bakke, T. Ramstad, and A. Hansen, arXiv:cond-mat/0507611 (unpublished). 